\title{
Skillful Sailors of Impossible Seas: Exploring Black Older Adults' Experience with Chronic Stress and Mental Health
}

Lauren Leigh Brown* is an assistant professor of public health at San Diego State University.

Brown, L. L. 2021. Skillful sailors of impossible seas: Exploring Black older adults' experience with chronic stress and mental health. Currents, (2)1.

Keywords: mental health

The term health paradox has fixed itself into popular discourse in public health, sociology, and demography as a way to describe counterintuitive findings related to health and social positioning. Take, for example, one of the hallmark health paradoxes: the Blackwhite mental health paradox. This refers to the unexplained phenomenon of Black Americans-despite experiencing more stress exposure, discrimination, and socioeconomic adversity, and as a result being in generally worse physical health-exhibiting lower rates of depressive and anxiety disorders relative to white Americans (Jackson et al., 2010; Mezuk et al., 2013; Williams, 2018; Williams \& Mohammed, 2008). Disproportionate exposure to stress and discrimination starts in early life (Liu et al., 2016; Thoits, 2010) and continues well into older adulthood for Black Americans (Brown

„brownll@umich.edu 
et al., 2018). Why then do Black people not always report higher rates of mental health disorders relative to whites? It may be that the Black-white paradox in mental health is an artifact of measurement error, implying existing anxiety and depression measures do not effectively capture the psychopathology of Black Americans (Aneshensel \& Mitchell, 2014; Takayanagi et al., 2014; Wetherell \& Areán, 1997). While there isn't enough empirical evidence to suggest that measurement error is the only culprit (Erving et al., 2018; Mezuk et al., 2013), psychopathology and diagnoses of psychiatric disorders have a long history of being centered and steeped in whiteness (Hunter \& Schmidt, 2010).

Still, the Black-white mental health paradox escapes easy explanation, but the assumption remains that white Americans should have better mental health than Black Americans based on differences in discrimination, stress exposure, education, income, and wealth. But data are painting a different picture. Social scientists, including James Jackson, David Williams, Lisa Bates, David Barnes, Dawn Mouzon, and Katherine Keyes, have spent their careers interrogating alternative hypotheses for the paradox. These hypotheses range from theories about Black-white differences in stress and coping (Brown et al., 2018; Keyes, 2009), protective factors (Mouzon, 2010, 2017), and health behaviors (Mezuk et al., 2013; Schnittker \& Do, 2020). James Jackson's environmental affordances model asserts that health behaviors-specifically alcohol and illicit drug use-in Black Americans lessens the effects of stress exposure on mental health while harming physical health (Mezuk et al., 2013). Dawne Mouzon (2010, 2017) tested hypotheses around race-specific protective factors like religiosity and social support that suggest that Black people have adapted coping mechanisms and other psychosocial resources that leave them better able to manage the mental health consequences of adversity. A recent study found that higher use of prescription medications that have negative metal health side effects account for higher rates of depression and suicide among white Americans (Schnittker \& Do, 2020). 
All of these hypotheses need more empirical support, yet the Blackwhite mental health paradox is a key empirical finding that suggests that factors other than stress exposure, discrimination, and socioeconomic adversity contribute to race differences in psychological well-being. In order to understand why Black Americans bear disproportionate stress exposure but similar or better mental health relative to whites, we consider race differences in not only stress exposure but also stress appraisal-how upsetting stress exposures are perceived to be (see Brown et al., 2020). Our study is novel for two reasons. First, instead of focusing on measurement error in mental health as an explanation for the paradox, we ask if there is something unique about the stress experience of Black Americans that is not captured by our general measures of stress exposure. Second, we explore evidence and explanations for the mental health paradox in anxiety and depression symptomology among older adults whom have faced a lifetime of chronic adversity. Black Americans may be better able to reframe their outlook on life, particularly in old age (Epel, 2009), affecting their psychological and behavioral responses to stress. Using data from the health and retirement study (HRS), we examine Black-white differences in stress exposure and appraisal on depressive and anxiety symptoms. The HRS is an ongoing community-based biennial study of diverse US adults age 51 and older that began in 1992, with the aim of improving our understanding of the social, economic, environmental, and behavioral factors associated with the health of older adults.

Building on the idea that the stress experience is different for older Black adults, we hypothesized that stress appraisal processes may inform our understanding of Black-white patterning in mental health. Our findings suggest stress theory has oversimplified the relationship between stress, socioeconomic disadvantage, discrimination, and mental health for Black older adults. First, Figure 1 shows older Black Americans report greater exposure to chronic stressors than older white Americans across health, housing, relationship, financial, and caregiving domains that undoubtedly have consequences for their mental health and well-being (Brown et al., 2020). 


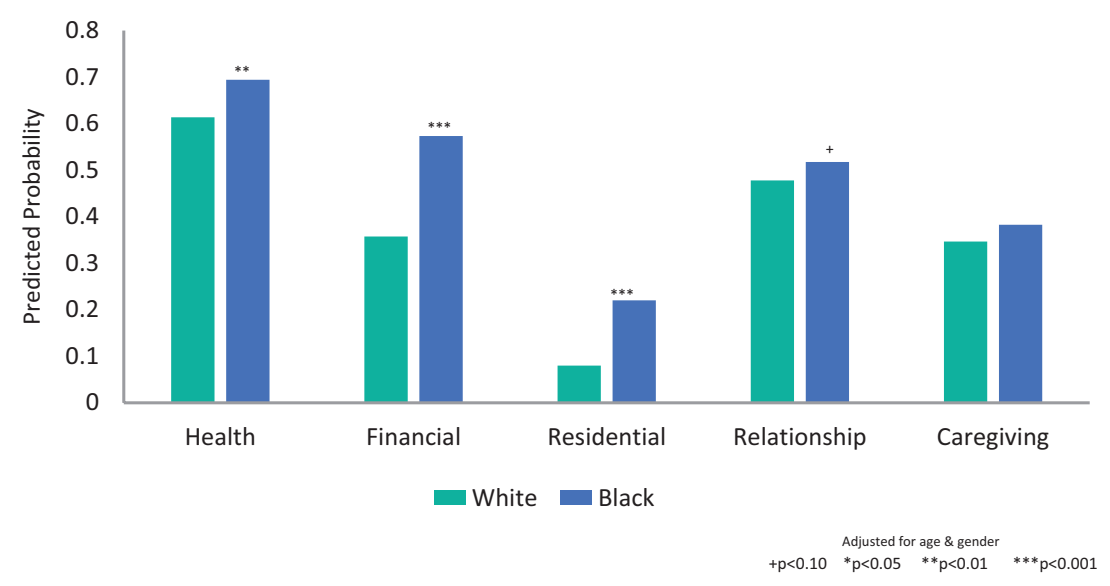

Figure 1. Black older adults more exposed to health, financial, residential strain, \& relationship strain

For both Black and white older adults, reporting greater exposure to chronic stress increases the likelihood of reporting more anxiety and depressive symptoms, especially when that stress exposure is considered to be upsetting for them. Yet there are notable race differences in stress exposure that place Black older adults at higher risk for reporting more anxiety and depressive symptoms relative to whites. For example, we establish an important link between financial and housing strain in late-life mental health. Black older adults are at least two times as likely to report facing financial and housing insecurity. These are severe and often unrelenting stressors patterned by social and structural disadvantages over the life course, capturing the most common hardships in later life for older Black adults (Brown et al., 2020). Several studies have documented broad psychological distress or depression among older adults who face financial and housing hardship (Angel et al., 2003; Kahn \& Pearlin, 2006). Older adults who have difficulty paying bills have been shown to delay or forego taking medications due to cost, which can have a devastating impact on mental health symptoms in addition to their physical health (Marshall et al., 2017, 2020). Experiencing financial or housing insecurity in later life poses a serious threat to the mental well-being of older Black adults. 


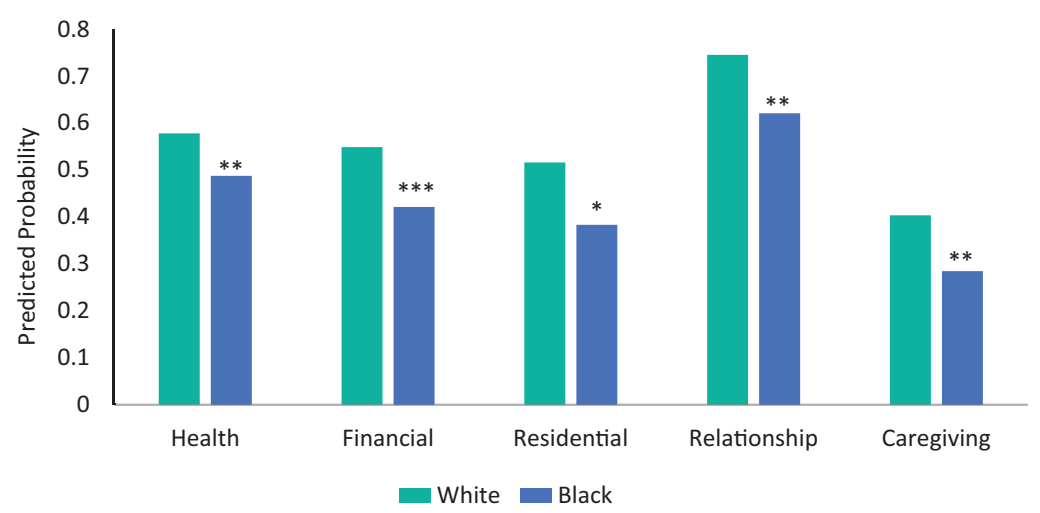

Adjusted for age, gender, education, income, wealth, marital \& employment status $+p<0.10 \quad * p<0.05 \quad * * p<0.01 \quad * * * p<0.001$

Figure 2. Black older adults less upset across every chronic stress domain

Yet, our findings also suggest that the degree to which a person appraises a stress exposure as a threat is crucial in determining the mental health consequences of stress exposure. Figure 2 shows that older Black adults generally appraise chronic stress exposure as less upsetting relative to older whites, suggesting they respond to stress differently, adapting to differential historical and current lived experiences.

We found stress appraisal independently predicts anxiety and depressive symptoms and it helps explain how Black-white differences in chronic stress exposure affect mental health symptomology. Stress appraisal moderates the interaction between race and stress exposure on anxiety symptoms and fully accounts for the interaction between race and stress exposure on depressive symptoms. Incorporating stress appraisal into disparity research may help us understand the similar or lower rates of depressive and anxiety disorders among Black Americans relative to white Americans despite large disparities in stress exposure and physical health in mid and late life. Stress appraisal is an overlooked pathway by which Black older adults may simultaneously report more exposure to stressors and adapt to that exposure, acknowledging the resourcefulness, resilience, agency, and effort Black Americans have used and are using to survive into older adulthood. 
Perhaps the African proverb is true: smooth seas do not make skillful sailors. Black older adults experience more stress but have also adapted ways to cope, some of which are likely intergenerational. Scholars increasingly recognize that the pathways to mental health unfold in different ways for Black and white Americans. Our findings suggest that these groups differ both in how much stress they experience and in how they respond to that stress. It may be that Black older adults have access to racially salient positive resources (i.e., religiosity, social support, racial identity) that may buffer the effects of stress on health. Although recent studies suggest that neither religious involvement (Mouzon, 2017), family relationships (Mouzon, 2010), nor relationships of choice (Mouzon, 2010) appear to explain the Black-white paradox in mental health, it is highly plausible that given a prolonged history of marginalization, older Black adults have developed unique coping mechanisms that may account for their mental hardiness.

The intersections of race and aging may also be an important factor by which older Black adults, over time, have developed astute coping mechanisms to buffer the effects of stress on mental health. Since Black older adults in our sample came of age during Jim Crow, desegregation, and the civil rights era, its plausible that they may perceive the chronic stressors that are measured in our study as less stressful since they have lived through very overt periods of racism and discrimination. This exposure to stress over the life course may have led them, as older adults, to strong adaptive mechanisms to help reframe stress or develop cognitive shifts to reduce the stressfulness of an exposure (Amirkhan, 1994; Harrell, 2000). Habituation, a theory originating in neurobiology, may be a particularly relevant stress response for older Black adults since the repetitiveness of chronic stress exposures over time lead to decreases in stress response or appraisals (Grissom \& Bhatnagar, 2009). Importantly, these hypotheses engage race and age-specific stress and coping mechanisms that highlight the distinct stress experience for older Black adults, a point that is relevant for all future Black-white paradox work. Future work 
will also need to explore at what point culturally based ways of coping no longer provide an effective way to deal with stress and mental health issues and become a barrier to accessing professional care.

Understanding the impact of chronic stress on the mental health of older adults represents an important frontier that is being magnified in the current collective global context. In the midst of the coronavirus pandemic, Black older adults have faced a plethora of uncertainties with respect to their health, worries which were exacerbated amid some of the isolation due to social distancing policies of the pandemic. The additional layer of economic uncertainty faced by many during the pandemic (Richter, 2020) necessitate solutions that alleviate financial insecurity and ensure adequate and stable housing for Black older adults. Financial security and stable housing are foundational resources and fundamental rights that older adults deserve access to. In order to fully address these chronic stressors, we also need to address the structural and institutional racism that perpetuates discrimination in access to housing and financial security. This may be one important mechanism by which we can directly address disparities in mental health for older adults.

\section{Biographies}

Lauren Brown, PhD, MPH her research aims to characterize not only the unique challenges Black and Brown people face in reaching older adulthood, but also making health injustices and scientific racism visible via data and storytelling. Email: Lbrown2@sdsu.edu. Twitter: @ profbrownnnn.

\section{References}

Amirkhan, J. H. (1994). Criterion validity of a coping measure. Journal of Personality Assessment, 62(2), 242-261. https://doi.org/10.1207/s15327752 jpa6202_6 
Aneshensel, C. S., \& Mitchell, U. A. (2014). The stress process. In W. C. Cockerham, R. Dingwall, \& S. Quah (Eds.), The Wiley Blackwell Encyclopedia of Health, Illness, Behavior, and Society (pp. 2334-2346). John Wiley \& Sons. https://doi.org/10.1002/9781118410868.wbehibs380

Angel, R. J., Frisco, M., Angel, J. L., \& Chiriboga, D. A. (2003). Financial strain and health among elderly Mexican-origin individuals. Journal of Health and Social Behavior, 44(4), 536. https://doi.org/10.2307/1519798

Brown, L. L., Abrams, L. R., Mitchell, U. A., \& Ailshire, J. A. (2020). Measuring more than exposure: Does stress appraisal matter for black-white differences in anxiety and depressive symptoms among older adults? Innovation in Aging, 4(5). https://doi.org/10.1093/geroni/igaa040

Brown, L. L., Mitchell, U. A., \& Ailshire, J. (2018). Disentangling the stress process: Race/ethnic differences in the exposure and appraisal of chronic stressors among older adults. The Journals of Gerontology: Series B, 65(3), gby072. https://doi.org/10.1093/geronb/gby072

Epel, E. S. (2009). Psychological and metabolic stress: A recipe for accelerated cellular aging? Hormones, 8(1), 7-22.

Erving, C. L., Thomas, C. S., \& Frazier, C. (2018). Is the black-white mental health paradox consistent across gender and psychiatric disorders? American Journal of Epidemiology, 188(2), 314-322. https://doi.org/10.1093/ aje/kwy224

Grissom, N., \& Bhatnagar, S. (2009). Habituation to repeated stress: Get used to it. Neurobiology of Learning and Memory, 92(2), 215-224. https://doi. org/10.1016/j.nlm.2008.07.001

Harrell, S. P. (2000). A multidimensional conceptualization of racism-related stress: Implications for the well-being of people of color. American Journal of Orthopsychiatry, 70(1), 42-57. https://doi.org/10.1037/h0087722

Hunter, L. R., \& Schmidt, N. B. (2010). Anxiety psychopathology in African American adults: Literature review and development of an empirically informed sociocultural model. Psychological Bulletin, 136(2), 211-235. https://doi.org/10.1037/a0018133

Jackson, J. S., Knight, K. M., \& Rafferty, J. A. (2010). Race and unhealthy behaviors: Chronic stress, the HPA axis, and physical and mental health disparities over the life course. American Journal of Public Health, 100(5), 933-939. https://doi.org/10.2105/AJPH.2008.143446

Kahn, J. R., \& Pearlin, L. I. (2006). Financial strain over the life course and health among older adults. Journal of Health and Social Behavior, 47(1), 17-31. https://doi.org/10.1177/002214650604700102

Keyes, C. L. M. (2009). The black-white paradox in health: Flourishing in the face of social inequality and discrimination: The black-white paradox in health. Journal of Personality, 77(6), 1677-1706. https://doi.org/10.1111/ j.1467-6494.2009.00597.x 
Liu, C. H., Giallo, R., Doan, S. N., Seidman, L. J., \& Tronick, E. (2016). Racial and ethnic differences in prenatal life stress and postpartum depression symptoms. Archives of Psychiatric Nursing, 30(1), 7-12. https://doi. org/10.1016/j.apnu.2015.11.002

Marshall, G. L., Kahana, E., Gallo, W. T., Stansbury, K. L., \& Thielke, S. (2020). The price of mental well-being in later life: The role of financial hardship and debt. Aging \& Mental Health, 1-7. https://doi.org/10.1080/13607863. 2020.1758902

Marshall, G. L., Thorpe, R. J., \& Szanton, S. L. (2017). Material hardship and self-rated mental health among older black Americans in the national survey of American life. Health \& Social Work, 42(2), 87-95. https://doi. org/10.1093/hsw/hlx008

Mezuk, B., Abdou, C. M., Hudson, D., Kershaw, K. N., Rafferty, J. A., Lee, H., \& Jackson, J. S. (2013). "White box" epidemiology and the social neuroscience of health behaviors: The environmental affordances model. Society and Mental Health, 3(2), 79-95. https://doi.org/10.1177/ 2156869313480892

Mouzon, D. (2010). Can social relationships explain the race paradox in mental health? [Doctoral dissertation, Rutgers, the State University of New Jersey]. Retrieved from https://rucore.libraries.rutgers.edu/rutgers-lib/ 30358/

Mouzon, D. (2017). Religious involvement and the black-white paradox in mental health. Race and Social Problems, 9(1), 63-78. https://doi. org/10.1007/s12552-017-9198-9

Richter, F. (2020, June 2). Impact of COVID-19 pandemic on mental health: Pandemic causes spike in anxiety \& depression. Statista. Retrieved from https://www.statista.com/chart/21878/impact-of-coronavirus-pandemicon-mental-health/

Schnittker, J., \& Do, D. (2020). Pharmaceutical side effects and mental health paradoxes among racial-ethnic minorities. Journal of Health and Social Behavior, 61(1), 3-3. https://doi.org/10.1177/0022146520903969

Takayanagi, Y., Spira, A. P., Roth, K. B., Gallo, J. J., Eaton, W. W., \& Mojtabai, R. (2014). Accuracy of reports of lifetime mental and physical disorders: Results from the Baltimore epidemiological catchment area study. JAMA Psychiatry, 71(3), 273. https://doi.org/10.1001/jamapsychiatry.2013.3579

Thoits, P. A. (2010). Stress and health: Major findings and policy implications. Journal of Health and Social Behavior, 51(1), S53. https://doi.org/ $10.1177 / 0022146510383499$

Wetherell, J. L., \& Areán, P. A. (1997). Psychometric evaluation of the Beck Anxiety Inventory with older medical patients. Psychological Assessment, 9(2), 136-144. https://doi.org/10.1037/1040-3590.9.2.136 
Williams, D. R. (2018). Stress and the mental health of populations of color: Advancing our understanding of race-related stressors. Journal of Health and Social Behavior, 59(4), 466-485. https://doi.org/10.1177/00221 46518814251

Williams, D. R., \& Mohammed, S. A. (2008). Discrimination and racial disparities in health: Evidence and needed research. Journal of Behavioral Medicine, 32(1), 20. https://doi.org/10.1007/s10865-008-9185-0 